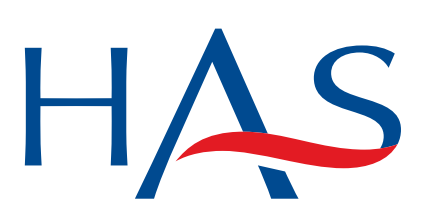

HAUTE AUTORITÉ DE SANTÉ

www.has-sante.fr

\title{
Are there differences between medicine appraisals in France in the paediatric versus the adult populations?
}

\author{
Chloé Rebstock', Jade Putzolu', Chantal Bélorgey², Jean-Christophe Mercier, Christian Thuillez ${ }^{3}$, Anne d'Andon \\ 1. Drug Assessment Department ; 2. Division of Medical, Economic and Public Health Assessment ; \\ 3. Transparency Committee. \\ French National Authority for Health, La Plaine Saint-Denis, France
}

\section{BACKGROUND AND AIMS}

The French National Authority for Health (HAS) is responsible for health technology assessment. The Transparency Committee (TC) of the HAS recommends the reimbursement of medicine by appraising two criteria:

- the clinical benefit (CB): assesses the intrinsic value of the drug
- 3 levels of "sufficient CB"
$\rightarrow$ positive recommendation of reimbursement
"important"/"moderate"/"mild"
- 1 level of "insufficient CB
$\rightarrow$ negative recommendation of reimbursement

- the clinical added value (CAV): assesses the comparative value of the drug in comparison to available treatments

- 5 levels $\rightarrow$ Taken into account in price negotiations

"major" (CAV I)/“important" (CAV II)/

"moderate" (CAV III)/"minor" (CAV IV)/“No CAV" (CAV V)

Hypothesis: more favourable TC assessment of paediatric drugs as compared to adult ones.

\section{METHODS}

\section{Retrospective study:}

1. Selection of TC appraisals concerning paediatric indications. Periods: - 2015-2017 for appraisal

- > 2010 for marketing authorization

2. Research of TC appraisals concerning adult for the same indication by medicine.

Periods: no limitation of date for appraisal and marketing authorization.

3. Comparison of appraisals between paediatric and adult.

$\rightarrow 116$ mixed indications (paediatric and adult) identified corresponding to 102 medicines

\section{RESULTS}

Concerning CB, appraisals were similar in 94\% (109/116) of the cases between paediatric and adult. CB was important in $90 \%(98 / 109)$ of the cases. (Figure 1).

\section{Figure 1. CB's appraisal:} paediatric vs adult ( $n=116$ indications)
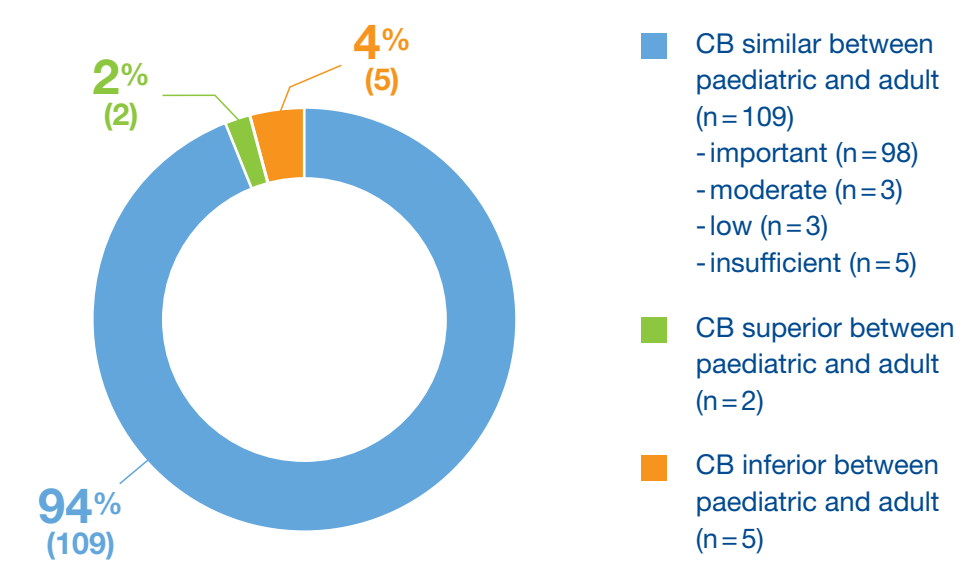

Discrepancies (7/116): $5 / 7$ in favour of adult ; $2 / 7$ in favour of paediatric (Table 1).

Table 1. Molecules and indications concerned by a different $C B$ ( $n=7 / 116$ indications)

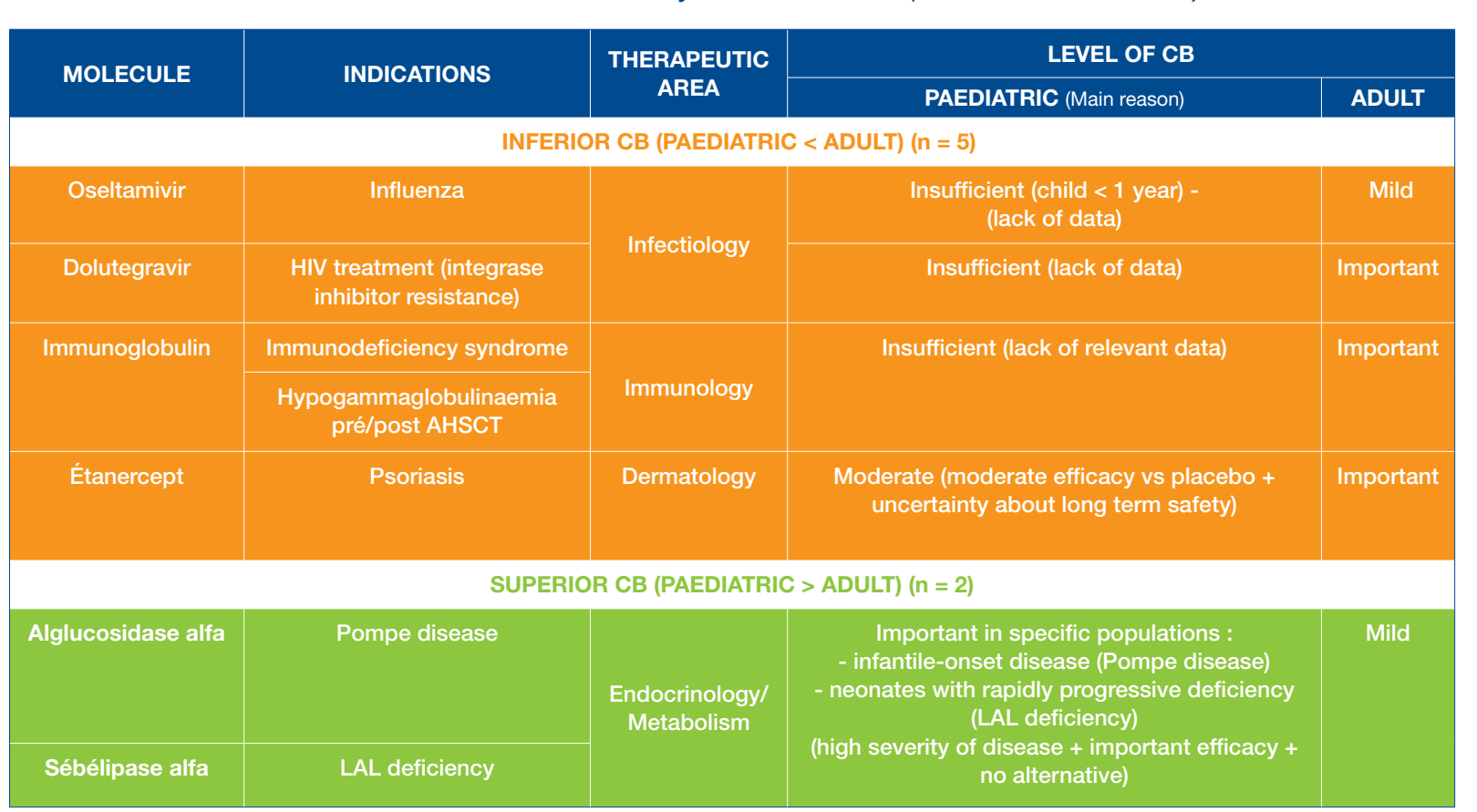

Concerning CAV, a comparison of appraisals was made for 105/116 indications (9 had an insufficient paediatric's CB and 2 adult's CAV were not available). CAV were similar in $90 \%(94 / 105)$ of the cases. "No CAV" was identified in $80 \%(75 / 94)$ of the cases (Figure 2).
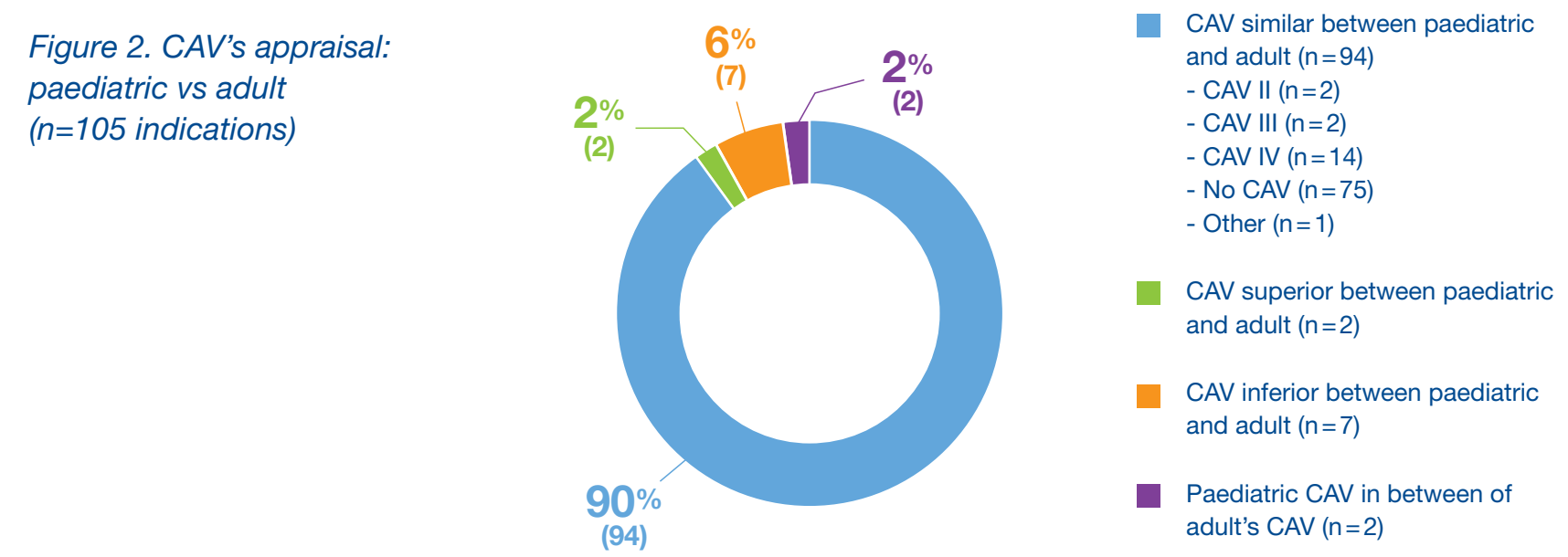

Discrepancies (11/105): 7/11 in favour of adult ; $2 / 11$ in favour of paediatric : 2/11 in beetween CAV (Table 2).

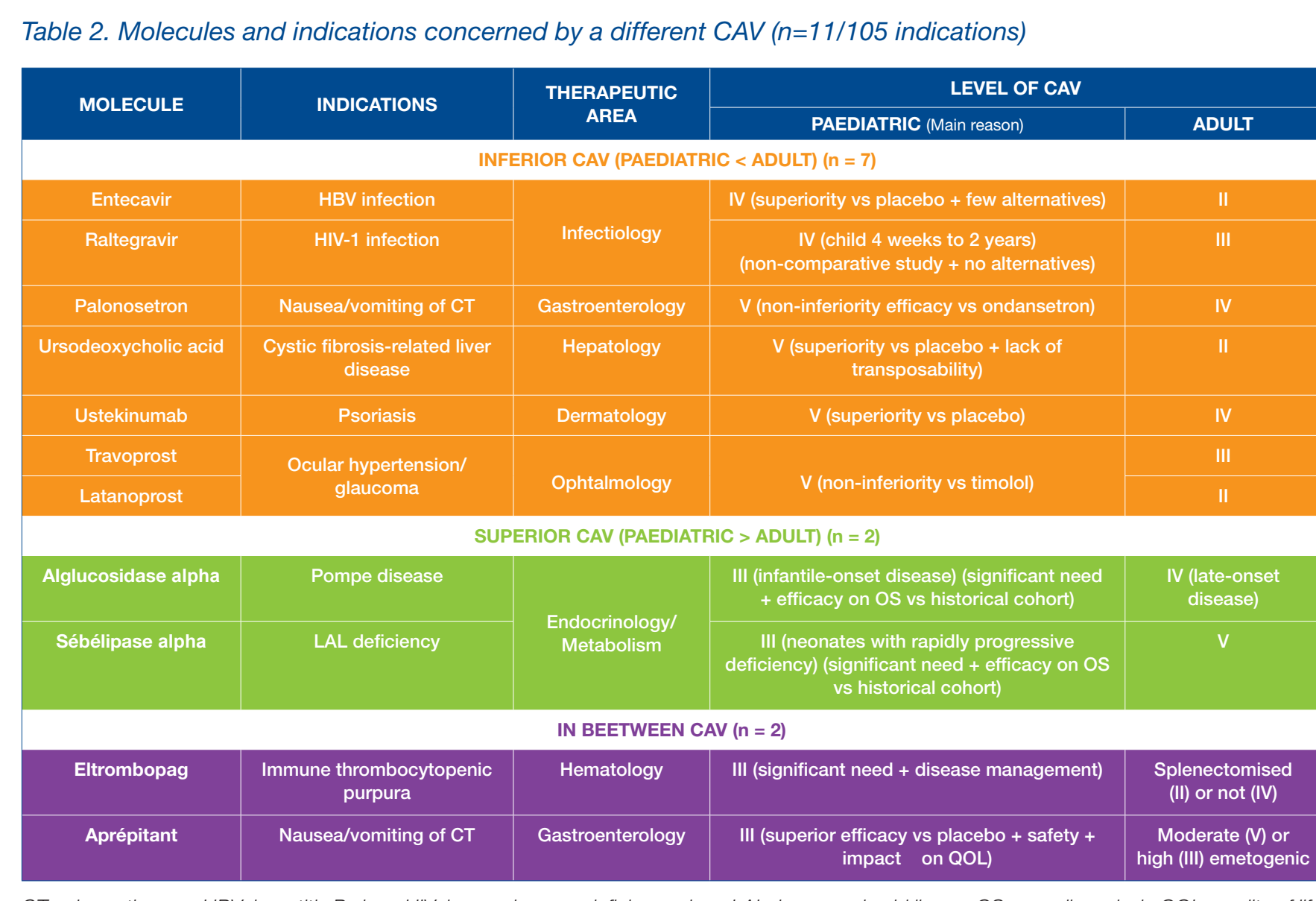

\section{CONCLUSIONS}

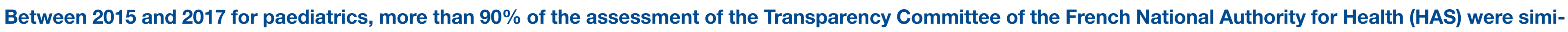

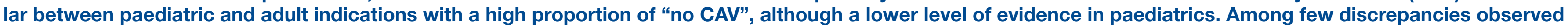

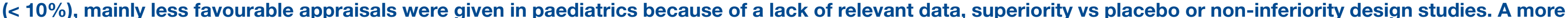

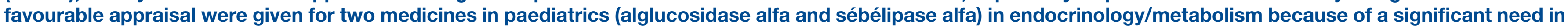

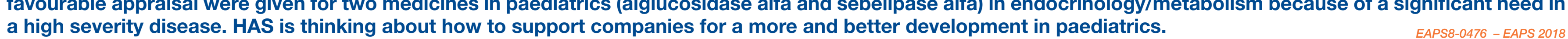

